\title{
APPLICATION OF THE PIV METHOD IN THE PRESENCE OF NEGATIVE DATA: AN EMPIRICAL EXAMPLE FROM A REAL-WORLD CASE
}

Nazlı ERSOY

Citation/C: Ersoy, N. (2021). Application of the PIV method in the presence of negative data: an empirical example from a real-world case. Hitit Journal of Social Sciences, 14(2), 318-337. doi: 10.17218/hititsbd.974522

Abstract: The presence of negative values in the decision matrix is a rare situation in Multiple Criteria Decision Making (MCDM) methods. In such a case, normalized matrix elements must be between 0 and 1 to use the Proximity Indexed Value (PIV) method. In this study, in which a real-life application is presented, two different solutions are generated for this problem. Firstly, negative decision matrix elements are converted to positive using the $Z$-score standardization method. Secondly, different normalization techniques are used instead of vector normalization in the algorithm of the PIV method. According to the obtained results, the most appropriate technique to use the PIV method in the presence of negative values in the decision matrix is the min-max normalization technique. The proposed model in this study supports the use the PIV method in the presence of negative values. In addition, this study is the first to test the suitability of different techniques for the PIV method.

Keywords: MCDM, PIV Method, Normalization, Standardization, Consistency

\section{Negatif Verilerin Varlığı Durumunda PIV Yönteminin Uygulanması: Gerçek Hayat Uygulamasina Dayalı Ampirik Bir Analiz}

Atıf/@): Ersoy, N. (2021). Negatif verilerin varlığı durumunda PIV yönteminin uygulanması: gerçek hayat uygulamasına dayalı ampirik bir analiz. Hitit Sosyal Bilimler Dergisi, 14(2), 318-337. doi: 10.17218/hititsbd.974522

Özet: Çok Kriterli Karar Verme (ÇKKV) yöntemlerinde karar matrisinde negatif verilere nadir olarak rastlanılmaktadır. Böyle bir durumda Proximity Indexed Value- Yakınlık Endeksli Değer (PIV) yöntemini uygulamak için normalize edilmiş karar matrisi elemanlarının 0 ile 1 arasında olacak şekilde yeniden düzenlenmesi gerekmektedir. Gerçek hayat uygulamasının ele alındığı bu çalışmada, mevcut problemi ortadan kaldırmak amacıyla iki farklı çözüm yolu sunulmuştur. İlk olarak, negatif karar matrisi elemanları, z-skor standardizasyon yöntemi kullanılarak pozitif hale getirilmiştir. İkinci olarak, PIV yönteminin algoritmasında bulunan vektör normalizasyon tekniği yerine farklı normalizasyon teknikleri kullanılmıştır. Elde edilen sonuçlara göre karar matrisinde negatif verilerin varlığ durumunda PIV yöntemi ile sonuca ulaşmak için en uygun teknik min-max tekniğidir. Bu çalışmada önerilen model, karar matrisinde negatif verilerin bulunması durumunda PIV yönteminin kullanımını desteklemektedir. Ayrıca, bu çalışma farklı tekniklerin PIV yöntemi için uygunluğunu test eden ilk çalışmadır.

Anahtar Kelimeler: ÇKKV, PIV Yöntemi, Normalizasyon, Standardizasyon, Tutarlilı 
Application of the PIV Method in the Presence of Negative Data: An Empirical Example from a Real-World Case

\section{INTRODUCTION}

Multiple Criteria Decision Making (MCDM) methods provide a suitable framework for decisionmakers to choose between multiple alternatives and multiple conflicting criteria. The basic algorithm of MCDM methods is based on criteria, alternative and criteria weights. Although the procedures of the methods differ from each other, there are many factors that affect MCDM results such as criteria weights, data set and the normalization process. The effect of different normalization procedures on MCDM results has been tackled by many studies (Kosareva et al. 2018; Vafaei et al. 2016; Yazdani et al. 2017a; Milani et al. 2005).

Normalized decision matrix elements will be a positive value between 0 and 1 (Yazdani et al., 2017a, p. 60). Negative values in the decision matrix are rarely seen in MCDM methods. Especially in MCDM methods where linear and vector normalization techniques are used, some problems are encountered when there are negative values in the normalized decision matrix. In this case, changing the normalization technique or standardizing the decision matrix and converting it to positive seem to be the two most important ways.

In this study, a real-life application is discussed by measuring the financial performances of 45 companies traded in the Borsa İstanbul (BIST) sustainability index with the Proximity Indexed Value (PIV) method. Firstly, negative decision matrix values are converted to positive using the $Z$ score standardization method developed by Zhang et al. (2014). As a second way, the PIV method steps were applied using enhanced accuracy and min-max normalization techniques instead of the vector normalization technique in the algorithm of the PIV method. In this study, a four-step process was applied to choose the most appropriate technique for the PIV method in presence of negative values in the decision matrix. In the first stage, Ranking Consistency Index (RCI) approach developed by Chakraborty and Yeh (2009), which takes into account the consistency between ranking results were used. In the second step, the approach suggested by Celen (2014) was considered and the Pearson correlation values of the ranking results obtained by different techniques were calculated. In the third step, the Standard Deviation (STD) of the ranking values was calculated by following the approach suggested by Bland and Altman (1996) and Yeh (2003). In the fourth step, distance measurements (Manhattan, Euclidean, Chebyshev) were calculated by following the approach suggested by Guo (2004) and Hassan et al. (2014). In the last step, the final result was obtained by using the plurality voting method.

The motivation and superiority of the proposed approach in this paper are outlined as follows:

- In this study, two different ways are proposed to use the PIV method in the presence of negative values in the decision matrix. Through the proposed model, different normalization techniques will be tested for the PIV method and the decision matrix will be standardized.

- This study is the first test of the suitability of different techniques for the PIV method in the presence of negative values in the decision matrix.

- This study guides the decision-makers to use the PIV method and other MCDM methods in the presence of negative values in the decision matrix.

The rest of the paper was organized as follows: In Section 2, the relevant previous literature studies are discussed. In Section 3, the mathematical notation and explanation of the methods used in the study are given. In Section 4, the application part of the study is included. Section 5 describes the proposed approach in this study to select the most suitable technique for the PIV method. Finally, concluding remarks and future research directions are included in Section 6. 


\section{LITERATURE REVIEW}

Many different topics such as health (Stevic et al. 2020), energy (Kumar et al. 2017), manufacturing (Abdel-Basset et al. 2020), banking (Dinçer and Yüksel, 2018), airline (Pineda et al. 2018), construction (Matic et al. 2019), material selection (Mousavi-Nasab and SotoudehAnvari, 2017), financial performance evaluation (Ghadikolaei et al. 2014), vendor selection (Shyur and Shih, 2006), supplier selection (Yazdani et al. 2017b), portfolio optimization (Ehrgott et al. 2004) were handled by using MCDM methods. Studies examining the effect of different normalization methods on MCDM results are given below.

Kosareva et al. (2018) used five different normalization techniques and they ranked the alternatives using the Simple Additive Weighting (SAW) method. It was concluded that none of the five methods are best or worst in all situations. However, the min-max normalization technique is significantly better than others in most cases. The Log method is the worst in some cases, but it is the best compared to other methods. Yazdani et al. (2017a) measured the effects of different normalization techniques for the COPRAS-G model. It was concluded that a different normalization tool would change the ranking results depending on the number of criteria and alternatives. Vafaei et al. (2016) measured the effects of different normalization techniques for the Analytical Hierarchy Process (AHP) method. At the end of the study, it was determined that the logarithmic normalization technique was not suitable for the AHP method. Jahan and Edwards (2015) examined the shortcomings of 18 different normalization techniques and made suggestions for their use in engineering. At the end of the study, it was determined that the differences between normalization techniques have a significant effect on the quality of decision making in material selection in engineering design. Vafaei et al. (2020) tested the suitability of five different normalization techniques for the AHP method. It was concluded that the max-min normalization technique was the most appropriate technique for the AHP, while the sum and vector normalization techniques were the least appropriate techniques. Milani et al. (2005) used the Entropy and the technique for order preference by similarity to ideal solution (TOPSIS) methods for selection the gear material and tested the suitability of five different normalization techniques. It was concluded that using both linear and non-linear norms is more suitable for engineering decisions.

Chakraborty and Yeh (2009) tested the suitability of four different normalization techniques for the TOPSIS method. At the end of the study, it was determined that the vector normalization technique is the most suitable for the TOPSIS method. Mathew et al. (2017) tested the suitability of different normalization techniques for the weighted aggregated sum product assessment (WASPAS) method. It was concluded that linear normalization (max-min) proved to be best normalization technique for the WASPAS method. Chakraborty and Yeh (2007) tested the suitability of four different normalization techniques for the SAW method. It was concluded that vector normalization, linear scale transformation and max method are more suitable for the SAW method. Celen (2014) evaluated the effects of the four normalization procedures on the TOPSIS method considering the financial performances of 13 Turkish deposit banks. It was concluded that the vector normalization procedure is the most convenient for the TOPSIS method. Vafaei et al. (2019) selected the better data normalization technique for supplier selecting problem. In the study where six different normalization techniques were used, a four-stage process was followed. Chatterjee and Chakraborty (2014) assessed the effect of different normalization techniques for the Preference Ranking Organization Method for Enrichment Evaluations (PROMETHEE), Grey Relation Analysis (GRA), TOPSIS methods. It was observed that the vector normalization procedure is the most preferred. 


\section{METHODS}

In this section, the methods used in the study are emphasized and mathematical notation and explanations are given.

\subsection{Normalization Techniques}

Normalization techniques can be classified in several ways. Only two of the normalization techniques presented in Table 1 will be used in this study. On the other hand, the max normalization technique (Stopp, 1975), the sum normalization technique (Wang and Luo, 2010), the logarithmic normalization technique (Zavadskas and Turskis, 2008), the Jüttler (1966) and Körth's (1969) normalization techniques, the Lai and Hwang normalization technique (Lai and Hwang, 1994), the peldschus normalisation technique (Peldschus, 1986), the Markovic normalization technique (Markovic, 2010), the linear normalization technique (Milani et al. 2005; Asgharpour, 1998; Farag, 1997; Tzeng and Huang, 2011), the vector normalization technique (Milani et al. 2005) could not be used since they lead to negative values in the normalized decision matrix. The normalization techniques used in this study are presented in Table 1.

Table 1. Normalization Techniques

\begin{tabular}{llcc}
\hline Normalization method & Source & Benefit criteria & Cost criteria \\
\hline $\begin{array}{l}\text { Enhanced accuracy } \\
\text { technique }\end{array}$ & $\begin{array}{l}\text { Zeng et al. } \\
(2013)\end{array}$ & $n_{i j}=1-\frac{r_{j}^{\max }-r_{i j}}{\sum_{i=1}^{m}\left(r_{j}^{\max }-r_{i j}\right)}$ & $n_{i j}=1-\frac{r_{i j}-r_{j}^{\min }}{\sum_{i=1}^{m}\left(r_{i j}-r_{j}^{\min }\right)}$ \\
\hline $\begin{array}{l}\text { Linear max min } \\
\text { normalization technique }\end{array}$ & $\begin{array}{l}\text { Weitendorf } \\
(1976)\end{array}$ & $n_{i j}=\frac{r_{i j}-r_{j}^{\min }}{r_{j}^{\max }-r_{j}^{\min }}$ & $n_{i j}=\frac{r_{j}^{\max }-r_{i j}}{r_{j}^{\max }-r_{j}^{\min }}$ \\
\hline
\end{tabular}

\subsection{Z-Score (Standard Score) Standardization Method}

In the evaluation process, decision matrix elements need to be standardized to compare criteria with different dimensions and units. On the other hand, the existence of negative value in the decision matrix is not frequently encountered in MCDM problems. In such a case, since the negative values cannot be included in the normalized matrix, the decision matrix elements must be converted to positive.

In this study, the Z-score standardization method which was introduced by Zhang et al. (2014) was used to turn negative data into positive. The steps of the $Z$-score standardization method are as follows (Zhang et al., 2014, p. 3).

Step 1: Decision matrix elements are transformed using equation (1).

$x_{i j}=\frac{X_{i j}-\overline{X_{l j}}}{S_{i}}$

$X_{i j}$ is the standardized data of the $i t h$ index in the $j$ th region

$X_{i j}$ is the original data

$X_{i}$ and $S_{i}$ are the mean value and standard deviation of the ith index.

Step 2: The negative elements of the decision matrix are converted to positive using equation (2). $x_{i j=}^{\prime} x_{i j}+A \quad A>\left|\min x_{i j}\right|$

$x_{i j}^{\prime}$ represents the standard value after transformation, $x_{i j}^{\prime}>0$

\subsection{PIV Method}

The PIV method was developed by Mufazzal and Muzakkir (2018). This method considers the proximity of alternatives to the best possible value. The steps of the PIV method are as follows (Mufazzal and Muzakkir, 2018, pp. 430-431): 
Step 1: Construction of decision matrix

In the first step, the decision matrix is created by determining the alternatives $A_{i}=(i=1,2,3, \ldots$, m) and criteria $C_{j}=(j=1,2,3, \ldots, n)$.

Step 2: Normalization of decision matrix

Decision matrix elements are normalized using equation (3).

$r_{i}=\frac{x_{i}}{\sqrt{\sum_{i=1}^{m} x_{i} 2}}$

$r_{i}$ is the actual decision value of the ith alternative.

Step 3: Determination of weighted normalized decision matrix

Each element in the normalized decision matrix is multiplied by the criterion weights using equation (4).

$v_{i=} w_{j} * r_{i}$

where $w_{j}$ is the weight of the jth criterion.

Step 4: Evaluation of Weighted Proximity Index (WPI)

WPI is calculated to find the closeness of each alternative to the best solution available.

$u_{i}=v_{\max }-v_{i}$ for benefit criteria

$u_{i}=v_{i}-v_{\min }$ for cost criteria

Step 5: Determination of overall proximity value

The total proximity value is calculated using equation (7).

$d_{i}=\sum_{j=1}^{n} u_{j}$

Step 6: Ranking of alternatives

The alternative with the lowest $d_{j}$ value represents the minimum deviation from the best solution and ranks first.

\section{APPLICATION}

The main purpose of this study is to decide whether the standardization of the decision matrix or changing the normalization technique is the right way to apply the PIV method in the presence of negative values in the decision matrix. In this study, the financial performances of 45 companies traded in the BİST sustainability index in 2019 were measured using the PIV method. The data was collected on June 2021 and was retrieved from the companies' financial reports. The alternatives and the criteria used in the study are presented in Appendix 1 and Table 2, respectively. The financial ratios determined by a comprehensive literature review were selected from among the ratios that provide information about the company's ability to pay its debts, the effective use of assets and resources, and profitability.

Table 2. Criteria

\begin{tabular}{|c|c|c|c|}
\hline Rank & Code & Financial Ratios and Disclosures & \\
\hline \multicolumn{3}{|c|}{ Liquidity ratios } & Opt. \\
\hline 1 & $\mathrm{CR}$ & Current ratio $=$ Current Assets / Current Liabilities & $\max$ \\
\hline 2 & QR & Quick ratio $=$ (Current Assets - Inventories) $/$ Current Liabilities & $\max$ \\
\hline \multicolumn{4}{|c|}{ Leverage ratios } \\
\hline 3 & DTE & Debt-to-Equity Ratio = Total Liabilities /Total Shareholders' Equity & $\min$ \\
\hline 4 & LR & Leverage Ratio $=$ Total Liabilities /Total assets & $\min$ \\
\hline \multicolumn{4}{|c|}{ Profitability ratios } \\
\hline 5 & ROE & Return on Equity = Net Income (annual)/ Shareholders' Equity & $\max$ \\
\hline 6 & ROA & Return on Assets = Net Income (annual) / Total assets & $\max$ \\
\hline \multicolumn{4}{|c|}{ Efficiency Ratios } \\
\hline 7 & WCT & Working capital turnover ratio $=$ Net sales $/$ Current Assets & $\max$ \\
\hline 8 & ATR & Asset Turnover Rate $=$ Net Sales $/$ Total Assets & $\max$ \\
\hline
\end{tabular}


Application of the PIV Method in the Presence of Negative Data:

An Empirical Example from a Real-World Case

\subsection{Weighting of Criteria}

Criterion weights are an important threshold in MCDM problems and have an impact on the results. In this study, the equal weighting technique developed by Jahan et al. (2012) was used to determine the criterion weights. The results obtained are presented in Table 3.

$w_{j}=\frac{1}{n}$

$\mathrm{n}$ indicates the number of criteria and the sum of weights should be equal to 1 .

Table 3. Criteria Weights

\begin{tabular}{rrrrrrrr}
\hline CR & QR & DTE & LR & ROE & ROA & WCTR & ATR \\
\hline 0.125 & 0.125 & 0.125 & 0.125 & 0.125 & 0.125 & 0.125 & 0.125 \\
\hline
\end{tabular}

\subsection{Standardization of the Decision Matrix Using Z-Score (Standard Score) Standardization Method}

Firstly, the decision matrix was created. The decision matrix with alternatives (firms) in rows and criteria (financial ratios) in columns is presented in Table 4.

Table 4. Decision Matrix

\begin{tabular}{|c|c|c|c|c|c|c|c|c|}
\hline \multirow{2}{*}{ Alternatives } & \multicolumn{8}{|l|}{ Criteria } \\
\hline & CR & QR & DTE & LR & ROE & ROA & WCTR & ATR \\
\hline AKENR & 0.52 & 0.52 & 6.15 & 0.86 & -0.77 & -0.11 & 3.38 & 0.12 \\
\hline AKSA & 1.05 & 0.73 & 1.73 & 0.63 & 0.18 & 0.07 & 1.67 & 0.87 \\
\hline AKSEN & 0.91 & 0.82 & 1.23 & 0.55 & 0.09 & 0.04 & 2.08 & 0.66 \\
\hline ANACM & 1.46 & 1.15 & 1.57 & 0.61 & 0.19 & 0.07 & 1.50 & 0.59 \\
\hline AEFES & 1.33 & 1.09 & 0.90 & 0.47 & 0.04 & 0.02 & 1.84 & 0.51 \\
\hline ANELE & 1.37 & 1.20 & 1.77 & 0.64 & 0.01 & 0.003 & 1.34 & 0.73 \\
\hline ARCLK & 1.58 & 1.19 & 2.54 & 0.72 & 0.10 & 0.03 & 1.44 & 0.96 \\
\hline ASELS & 1.80 & 1.23 & 0.89 & 0.47 & 0.25 & 0.13 & 0.93 & 0.51 \\
\hline AYGAZ & 1.06 & 0.89 & 1.00 & 0.50 & 0.11 & 0.06 & 6.43 & 2.06 \\
\hline BRISA & 0.95 & 0.68 & 4.89 & 0.83 & 0.13 & 0.02 & 1.60 & 0.67 \\
\hline CCOLA & 1.55 & 1.30 & 1.17 & 0.54 & 0.13 & 0.06 & 2.24 & 0.77 \\
\hline CIMSA & 0.78 & 0.65 & 1.54 & 0.61 & 0.01 & 0.003 & 1.64 & 0.46 \\
\hline DOHOL & 2.47 & 2.25 & 0.47 & 0.32 & 0.08 & 0.05 & 1.90 & 1.18 \\
\hline DOAS & 0.81 & 0.51 & 2.72 & 0.73 & 0.06 & 0.02 & 3.93 & 2.12 \\
\hline ENJSA & 0.79 & 0.77 & 2.42 & 0.71 & 0.15 & 0.04 & 3.36 & 0.83 \\
\hline ENKAI & 3.00 & 2.73 & 0.25 & 0.20 & 0.10 & 0.08 & 0.62 & 0.22 \\
\hline EREGL & 2.55 & 1.57 & 0.49 & 0.33 & 0.11 & 0.07 & 1.14 & 0.59 \\
\hline FROTO & 1.17 & 0.96 & 2.52 & 0.72 & 0.42 & 0.12 & 3.86 & 2.39 \\
\hline GLYHO & 0.86 & 0.80 & 3.65 & 0.78 & -0.09 & -0.02 & 1.03 & 0.20 \\
\hline SAHOL & 0.74 & 0.73 & 5.11 & 0.84 & 0.06 & 0.01 & 0.09 & 0.50 \\
\hline ISDMR & 3.32 & 1.96 & 0.34 & 0.25 & 0.13 & 0.10 & 1.68 & 0.76 \\
\hline KERVT & 2.47 & 1.80 & 1.82 & 0.65 & 0.14 & 0.05 & 1.63 & 0.92 \\
\hline KCHOL & 1.39 & 1.10 & 2.05 & 0.67 & 0.09 & 0.03 & 1.86 & 1.01 \\
\hline KORDS & 1.18 & 0.71 & 1.54 & 0.61 & 0.12 & 0.05 & 1.67 & 0.72 \\
\hline LOGO & 1.55 & 1.55 & 0.97 & 0.49 & 0.22 & 0.11 & 1.12 & 0.54 \\
\hline MGROS & 0.70 & 0.35 & 43.79 & 0.98 & -1.52 & -0.03 & 4.39 & 1.60 \\
\hline OTKAR & 1.87 & 1.13 & 3.20 & 0.76 & 0.55 & 0.13 & 1.25 & 0.91 \\
\hline PETKIM & 1.86 & 1.68 & 2.05 & 0.67 & 0.17 & 0.05 & 1.22 & 0.79 \\
\hline POLHO & 1.32 & 1.24 & 0.59 & 0.37 & 0.05 & 0.03 & 1.64 & 0.44 \\
\hline SODA & 2.66 & 2.30 & 0.50 & 0.33 & 0.19 & 0.13 & 1.03 & 0.48 \\
\hline TATGD & 2.84 & 1.61 & 0.83 & 0.45 & 0.12 & 0.06 & 1.46 & 1.11 \\
\hline TAVHL & 1.20 & 1.19 & 1.93 & 0.66 & 0.06 & 0.02 & 0.66 & 0.19 \\
\hline TKFEN & 1.39 & 1.17 & 1.30 & 0.57 & 0.26 & 0.11 & 1.58 & 1.15 \\
\hline TOASO & 1.14 & 1.02 & 1.96 & 0.66 & 0.34 & 0.12 & 2.71 & 1.48 \\
\hline TRKCM & 1.81 & 1.44 & 1.19 & 0.54 & 0.11 & 0.05 & 0.96 & 0.45 \\
\hline TCELL & 1.53 & 1.51 & 1.53 & 0.60 & 0.18 & 0.07 & 1.24 & 0.52 \\
\hline TUPRS & 0.99 & 0.66 & 3.23 & 0.76 & 0.04 & 0.01 & 3.21 & 1.61 \\
\hline THYAO & 0.80 & 0.75 & 2.60 & 0.72 & 0.11 & 0.03 & 2.65 & 0.51 \\
\hline TTKOM & 0.88 & 0.86 & 3.23 & 0.76 & 0.25 & 0.06 & 1.96 & 0.59 \\
\hline TTRAK & 1.77 & 1.19 & 2.82 & 0.74 & 0.15 & 0.04 & 1.97 & 1.31 \\
\hline SISE & 2.10 & 1.64 & 1.03 & 0.51 & 0.10 & 0.05 & 1.05 & 0.47 \\
\hline ULKER & 1.28 & 1.20 & 1.59 & 0.61 & 0.18 & 0.07 & 0.89 & 0.61 \\
\hline VESBE & 1.06 & 0.84 & 1.76 & 0.64 & 0.31 & 0.11 & 2.20 & 1.40 \\
\hline VESTL & 0.66 & 0.46 & 4.15 & 0.81 & 0.09 & 0.02 & 1.79 & 0.88 \\
\hline ZOREN & 0.51 & 0.50 & 10.39 & 0.91 & -0.08 & -0.01 & 1.96 & 0.45 \\
\hline
\end{tabular}


Step 1: Decision matrix with negative values is transformed using equation (1) and the results are presented in Table 5.

Table 5. Z-Score Standardization

\begin{tabular}{|c|c|c|c|c|c|c|c|c|}
\hline Alternatives & $\mathbf{C R}$ & QR & DTE & LR & ROE & ROA & WCTR & ATR \\
\hline AKENR & -0.764 & -1.206 & 0.472 & 1.396 & -2.824 & -3.323 & 1.287 & -1.357 \\
\hline AKSA & 0.202 & -0.802 & -0.212 & 0.073 & 0.323 & 0.427 & -0.207 & 0.075 \\
\hline AKSEN & -0.793 & -0.629 & -0.289 & -0.387 & 0.025 & -0.198 & 0.151 & -0.326 \\
\hline ANACM & 0.187 & 0.006 & -0.236 & -0.042 & 0.356 & 0.427 & -0.355 & -0.460 \\
\hline AEFES & -0.058 & -0.110 & -0.340 & -0.847 & -0.141 & -0.615 & -0.058 & -0.613 \\
\hline ANELE & -0.303 & 0.102 & -0.206 & 0.131 & -0.250 & -0.969 & -0.495 & -0.193 \\
\hline ARCLK & -0.317 & 0.083 & -0.086 & 0.591 & 0.058 & -0.406 & -0.408 & 0.246 \\
\hline ASELS & 1.067 & 0.160 & -0.342 & -0.847 & 0.554 & 1.677 & -0.853 & -0.613 \\
\hline AYGAZ & 0.159 & -0.495 & -0.325 & -0.675 & 0.091 & 0.219 & 3.951 & 2.346 \\
\hline BRISA & -0.865 & -0.899 & 0.277 & 1.223 & 0.157 & -0.615 & -0.268 & -0.307 \\
\hline CCOLA & 1.110 & 0.294 & -0.298 & -0.445 & 0.157 & 0.219 & 0.291 & -0.116 \\
\hline CIMSA & -2.436 & -0.956 & -0.241 & -0.042 & -0.240 & -0.969 & -0.233 & -0.708 \\
\hline DOHOL & 2.393 & 2.122 & -0.407 & -1.710 & -0.009 & 0.010 & -0.006 & 0.666 \\
\hline DOAS & 0.029 & -1.226 & -0.059 & 0.648 & -0.075 & -0.615 & 1.767 & 2.461 \\
\hline ENJSA & -3.185 & -0.725 & -0.105 & 0.533 & 0.223 & -0.198 & 1.269 & -0.002 \\
\hline ENKAI & 0.649 & 3.046 & -0.441 & -2.400 & 0.058 & 0.635 & -1.124 & -1.166 \\
\hline EREGL & 1.989 & 0.814 & -0.404 & -1.652 & 0.091 & 0.427 & -0.670 & -0.460 \\
\hline FROTO & 0.447 & -0.360 & -0.089 & 0.591 & 1.118 & 1.469 & 1.706 & 2.976 \\
\hline GLYHO & 0.173 & -0.668 & 0.085 & 0.936 & -0.572 & -1.448 & -0.766 & -1.204 \\
\hline SAHOL & -3.719 & -0.802 & 0.311 & 1.281 & -0.075 & -0.823 & -1.587 & -1.491 \\
\hline ISDMR & 1.225 & 1.564 & -0.427 & -2.112 & 0.157 & 1.052 & -0.198 & -0.135 \\
\hline KERVT & 1.557 & 1.256 & -0.198 & 0.188 & 0.190 & 0.010 & -0.242 & 0.170 \\
\hline KCHOL & 0.303 & -0.090 & -0.162 & 0.303 & 0.025 & -0.406 & -0.041 & 0.342 \\
\hline KORDS & -0.533 & -0.841 & -0.241 & -0.042 & 0.124 & 0.010 & -0.207 & -0.212 \\
\hline LOGO & 1.225 & 0.775 & -0.329 & -0.732 & 0.455 & 1.260 & -0.687 & -0.555 \\
\hline MGROS & -1.686 & -1.534 & 6.298 & 2.086 & $-5.308^{*}$ & -1.656 & 2.169 & 1.468 \\
\hline OTKAR & 0.014 & -0.033 & 0.016 & 0.821 & 1.548 & 1.677 & -0.574 & 0.151 \\
\hline PETKIM & 0.778 & 1.026 & -0.162 & 0.303 & 0.289 & 0.010 & -0.600 & -0.078 \\
\hline POLHO & -1.931 & 0.179 & -0.388 & -1.422 & -0.108 & -0.406 & -0.233 & -0.746 \\
\hline SODA & -0.259 & 2.219 & -0.402 & -1.652 & 0.356 & 1.677 & -0.766 & -0.670 \\
\hline TATGD & 2.364 & 0.891 & -0.351 & -0.962 & 0.124 & 0.219 & -0.390 & 0.533 \\
\hline TAVHL & -0.274 & 0.083 & -0.181 & 0.246 & -0.075 & -0.615 & -1.089 & -1.223 \\
\hline TKFEN & 0.360 & 0.044 & -0.278 & -0.272 & 0.588 & 1.260 & -0.285 & 0.609 \\
\hline TOASO & -0.966 & -0.244 & -0.176 & 0.246 & 0.853 & 1.469 & 0.702 & 1.239 \\
\hline TRKCM & 0.404 & 0.564 & -0.295 & -0.445 & 0.091 & 0.010 & -0.827 & -0.727 \\
\hline TCELL & 0.778 & 0.698 & -0.243 & -0.099 & 0.323 & 0.427 & -0.582 & -0.593 \\
\hline TUPRS & 0.274 & -0.937 & 0.020 & 0.821 & -0.141 & -0.823 & 1.138 & 1.487 \\
\hline THYAO & -0.115 & -0.764 & -0.077 & 0.591 & 0.091 & -0.406 & 0.649 & -0.613 \\
\hline TTKOM & -1.283 & -0.552 & 0.020 & 0.821 & 0.554 & 0.219 & 0.047 & -0.460 \\
\hline TTRAK & -0.476 & 0.083 & -0.043 & 0.706 & 0.223 & -0.198 & 0.055 & 0.914 \\
\hline SISE & 1.182 & 0.949 & -0.320 & -0.617 & 0.058 & 0.010 & -0.748 & -0.689 \\
\hline ULKER & 0.317 & 0.102 & -0.233 & -0.042 & 0.323 & 0.427 & -0.888 & -0.422 \\
\hline VESBE & 0.577 & -0.591 & -0.207 & 0.131 & 0.753 & 1.260 & 0.256 & 1.086 \\
\hline VESTL & 0.216 & -1.322 & 0.163 & 1.108 & 0.025 & -0.615 & -0.102 & 0.094 \\
\hline ZOREN & 0.735 & -1.245 & 1.129 & 1.683 & -0.539 & -1.240 & 0.047 & -0.727 \\
\hline
\end{tabular}

${ }^{*}$ The A value in Equation (1.2) has been taken as 5.3874803 
Application of the PIV Method in the Presence of Negative Data: An Empirical Example from a Real-World Case

Step 2: Negative values in the decision matrix are converted to positive using equation (2). The results are presented in Table 6.

Table 6. Positive Decision Matrix

\begin{tabular}{|c|c|c|c|c|c|c|c|c|}
\hline Alternatives & CR & QR & DTE & LR & ROE & ROA & WCTR & ATR \\
\hline AKENR & 4.624 & 4.181 & 5.860 & 6.783 & 2.563 & 2.065 & 6.674 & 4.031 \\
\hline AKSA & 5.589 & 4.585 & 5.176 & 5.461 & 5.710 & 5.815 & 5.181 & 5.462 \\
\hline AKSEN & 4.595 & 4.758 & 5.098 & 5.001 & 5.412 & 5.190 & 5.539 & 5.061 \\
\hline ANACM & 5.575 & 5.393 & 5.151 & 5.346 & 5.743 & 5.815 & 5.032 & 4.928 \\
\hline AEFES & 5.330 & 5.278 & 5.047 & 4.540 & 5.246 & 4.773 & 5.329 & 4.775 \\
\hline ANELE & 5.085 & 5.489 & 5.182 & 5.518 & 5.137 & 4.419 & 4.893 & 5.195 \\
\hline ARCLK & 5.070 & 5.470 & 5.301 & 5.978 & 5.445 & 4.981 & 4.980 & 5.634 \\
\hline ASELS & 6.454 & 5.547 & 5.046 & 4.540 & 5.942 & 7.065 & 4.534 & 4.775 \\
\hline AYGAZ & 5.546 & 4.893 & 5.063 & 4.713 & 5.478 & 5.606 & 9.338 & 7.734 \\
\hline BRISA & 4.523 & 4.489 & 5.665 & 6.611 & 5.544 & 4.773 & 5.120 & 5.080 \\
\hline CCOLA & 6.497 & 5.682 & 5.089 & 4.943 & 5.544 & 5.606 & 5.679 & 5.271 \\
\hline CIMSA & 2.952 & 4.431 & 5.146 & 5.346 & 5.147 & 4.419 & 5.155 & 4.680 \\
\hline DOHOL & 7.780 & 7.510 & 4.981 & 3.678 & 5.379 & 5.398 & 5.382 & 6.054 \\
\hline DOAS & 5.416 & 4.162 & 5.329 & 6.036 & 5.313 & 4.773 & 7.155 & 7.848 \\
\hline ENJSA & 2.202 & 4.662 & 5.283 & 5.921 & 5.611 & 5.190 & 6.657 & 5.386 \\
\hline ENKAI & 6.036 & 8.433 & 4.947 & 2.988 & 5.445 & 6.023 & 4.264 & 4.221 \\
\hline EREGL & 7.377 & 6.201 & 4.984 & 3.735 & 5.478 & 5.815 & 4.718 & 4.928 \\
\hline FROTO & 5.834 & 5.028 & 5.298 & 5.978 & 6.505 & 6.856 & 7.094 & 8.363 \\
\hline GLYHO & 5.560 & 4.720 & 5.473 & 6.323 & 4.816 & 3.940 & 4.622 & 4.183 \\
\hline SAHOL & 1.669 & 4.585 & 5.699 & 6.668 & 5.313 & 4.565 & 3.801 & 3.897 \\
\hline ISDMR & 6.613 & 6.952 & 4.961 & 3.275 & 5.544 & 6.440 & 5.189 & 5.252 \\
\hline KERVT & 6.944 & 6.644 & 5.190 & 5.576 & 5.578 & 5.398 & 5.146 & 5.558 \\
\hline KCHOL & 5.690 & 5.297 & 5.225 & 5.691 & 5.412 & 4.981 & 5.347 & 5.729 \\
\hline KORDS & 4.854 & 4.547 & 5.146 & 5.346 & 5.511 & 5.398 & 5.181 & 5.176 \\
\hline LOGO & 6.613 & 6.163 & 5.058 & 4.655 & 5.843 & 6.648 & 4.700 & 4.832 \\
\hline MGROS & 3.701 & 3.854 & 11.685 & 7.473 & 0.079 & 3.731 & 7.556 & 6.856 \\
\hline OTKAR & 5.402 & 5.355 & 5.403 & 6.208 & 6.936 & 7.065 & 4.814 & 5.538 \\
\hline PETKIM & 6.166 & 6.413 & 5.225 & 5.691 & 5.677 & 5.398 & 4.788 & 5.309 \\
\hline POLHO & 3.456 & 5.566 & 4.999 & 3.965 & 5.279 & 4.981 & 5.155 & 4.641 \\
\hline SODA & 5.128 & 7.606 & 4.985 & 3.735 & 5.743 & 7.065 & 4.622 & 4.718 \\
\hline TATGD & 7.751 & 6.278 & 5.036 & 4.425 & 5.511 & 5.606 & 4.997 & 5.920 \\
\hline TAVHL & 5.114 & 5.470 & 5.207 & 5.633 & 5.313 & 4.773 & 4.299 & 4.164 \\
\hline TKFEN & 5.748 & 5.432 & 5.109 & 5.115 & 5.975 & 6.648 & 5.102 & 5.997 \\
\hline TOASO & 4.422 & 5.143 & 5.211 & 5.633 & 6.240 & 6.856 & 6.089 & 6.626 \\
\hline TRKCM & 5.791 & 5.951 & 5.092 & 4.943 & 5.478 & 5.398 & 4.561 & 4.660 \\
\hline TCELL & 6.166 & 6.086 & 5.145 & 5.288 & 5.710 & 5.815 & 4.805 & 4.794 \\
\hline TUPRS & 5.661 & 4.450 & 5.408 & 6.208 & 5.246 & 4.565 & 6.526 & 6.875 \\
\hline THYAO & 5.272 & 4.624 & 5.310 & 5.978 & 5.478 & 4.981 & 6.037 & 4.775 \\
\hline TTKOM & 4.105 & 4.835 & 5.408 & 6.208 & 5.942 & 5.606 & 5.434 & 4.928 \\
\hline TTRAK & 4.912 & 5.470 & 5.344 & 6.093 & 5.611 & 5.190 & 5.443 & 6.302 \\
\hline SISE & 6.569 & 6.336 & 5.067 & 4.770 & 5.445 & 5.398 & 4.639 & 4.699 \\
\hline ULKER & 5.705 & 5.489 & 5.154 & 5.346 & 5.710 & 5.815 & 4.499 & 4.966 \\
\hline VESBE & 5.964 & 4.797 & 5.180 & 5.518 & 6.141 & 6.648 & 5.644 & 6.474 \\
\hline VESTL & 5,604 & 4.066 & 5.550 & 6.496 & 5.412 & 4.773 & 5.286 & 5.481 \\
\hline ZOREN & 6.123 & 4.143 & 6.516 & 7.071 & 4.849 & 4.148 & 5.434 & 4.660 \\
\hline
\end{tabular}




\subsection{Evaluation of Firms' Performance using the PIV Method}

In this section, the positive decision matrix in Table 6 will be used to apply the PIV method. In the first step, the elements in in Table 6 are normalized using equation (3). In the second step, the weighted normalized matrix was obtained by multiplying the normalized criteria values and the criteria weights in Table 3. Then, considering the benefit-oriented (CR, QR, ROE, ROA, WCTR, ATR) and cost-oriented criteria (DTE, LR), the deviation of each alternative from the best value was measured using equation (5) and the sum of elements in each row was calculated using equation (6). The results are presented in Table 7.

Table 7. Ranking Results Obtained by the PIV Method

\begin{tabular}{lrrlrrlrr}
\hline Firms & $\sum$ & Rank & Firms & $\sum$ & Rank & Firms & $\sum$ & Rank \\
\hline AKENR & 0.097 & 43 & ENKAI & 0.046 & 6 & TATGD & 0.046 & 5 \\
\hline AKSA & 0.062 & 24 & EREGL & 0.048 & 8 & TAVHL & 0.074 & 38 \\
\hline AKSEN & 0.066 & 29 & FROTO & 0.039 & 3 & TKFEN & 0.052 & 12 \\
\hline ANACM & 0.061 & 21 & GLYHO & 0.081 & 41 & TOASO & 0.052 & 13 \\
\hline AEFES & 0.064 & 28 & SAHOL & 0.097 & 44 & TRKCM & 0.062 & 22 \\
\hline ANELE & 0.070 & 34 & ISDMR & 0.042 & 4 & TCELL & 0.058 & 19 \\
\hline ARCLK & 0.067 & 30 & KERVT & 0.053 & 14 & TUPRS & 0.062 & 23 \\
\hline ASELS & 0.052 & 11 & KCHOL & 0.063 & 26 & THYAO & 0.068 & 33 \\
\hline AYGAZ & 0.038 & 1 & KORDS & 0.067 & 31 & TTKOM & 0.070 & 35 \\
\hline BRISA & 0.077 & 39 & LOGO & 0.051 & 9 & TTRAK & 0.063 & 27 \\
\hline CCOLA & 0.054 & 15 & MGROS & 0.113 & 45 & SISE & 0.057 & 18 \\
\hline CIMSA & 0.080 & 40 & OTKAR & 0.056 & 16 & ULKER & 0.062 & 25 \\
\hline DOHOL & 0.038 & 2 & PETKIM & 0.058 & 20 & VESBE & 0.051 & 10 \\
\hline DOAS & 0.057 & 17 & POLHO & 0.067 & 32 & VESTL & 0.073 & 36 \\
\hline ENJSA & 0.073 & 37 & SODA & 0.047 & 7 & ZOREN & 0.082 & 42 \\
\hline
\end{tabular}

\subsection{Application of Different Normalization Methods}

At this stage enhanced accuracy and linear max-min normalization techniques were used instead of the vector normalization technique in the PIV methods' algorithm. Table 8 presents an example of normalization calculation using the AKENR's benefit criteria (CR) and AKENR's cost criteria (DTE).

Table 8. Normalization Sample

\begin{tabular}{|c|c|c|c|c|}
\hline $\begin{array}{l}\text { Normalization } \\
\text { technique }\end{array}$ & $\begin{array}{l}\text { Condition } \\
\text { of use }\end{array}$ & Formula & Process & Value \\
\hline \multirow{4}{*}{$\begin{array}{l}\text { Enhanced } \\
\text { accuracy } \\
\text { technique }\end{array}$} & \multirow[b]{2}{*}{$\begin{array}{l}\text { Benefit } \\
\text { criteria }\end{array}$} & \multirow{2}{*}{$n_{i j}=1-\frac{r_{j}^{\max }-r_{i j}}{\sum_{i=1}^{m}\left(r_{j}^{\max }-r_{i j}\right)}$} & & \multirow{2}{*}{0.967} \\
\hline & & & $1-\frac{3.32-0.52}{\sum^{m}(3.32-0.52)+(3.32-1.05)+\ldots+(3}$ & \\
\hline & \multirow[b]{2}{*}{ Cost criteria } & $n_{i j}=1-\frac{r_{i j}-r_{j}^{\min }}{m}$ & $6.15-0.25$ & \multirow[b]{2}{*}{0.954} \\
\hline & & $n_{i j}=1-\frac{l}{\sum_{i=1}^{m}\left(r_{i j}-r_{j}^{\min }\right)}$ & $\sum_{i=1}^{m}(6.15-0.25)+(1.73-0.25)+\ldots+(10.39-0.25)$ & \\
\hline \multirow{4}{*}{$\begin{array}{l}\text { Linear max min } \\
\text { normalization } \\
\text { technique }\end{array}$} & \multirow{2}{*}{$\begin{array}{l}\text { Benefit } \\
\text { criteria }\end{array}$} & \multirow{2}{*}{$n_{i j}=\frac{r_{i j}-r_{j}^{\min }}{r_{j}^{\max }-r_{j}^{\min }}$} & $0.52-0.51$ & \multirow[t]{2}{*}{0.004} \\
\hline & & & $\overline{3.32-0.51}$ & \\
\hline & \multirow{2}{*}{ Cost criteria } & \multirow{2}{*}{$n_{i j}=\frac{r_{j}^{\max }-r_{i j}}{r_{j}^{\max }-r_{j}^{\min }}$} & $43.79-6.15$ & \multirow[t]{2}{*}{0.864} \\
\hline & & & $\overline{43.79-0.25}$ & \\
\hline
\end{tabular}

The normalization process shown in Table 8 has been applied to all criteria. The ranking results obtained are given in Table 9. 
Application of the PIV Method in the Presence of Negative Data: An Empirical Example from a Real-World Case

Table 9. Final Ranking Results Obtained with Three Different Techniques

\begin{tabular}{|c|c|c|c|}
\hline & Z-Score & Enhanced accuracy & Min-max \\
\hline & Rank & Rank & Rank \\
\hline AKENR & 43 & 45 & 45 \\
\hline AKSA & 24 & 32 & 31 \\
\hline AKSEN & 29 & 40 & 37 \\
\hline ANACM & 21 & 25 & 27 \\
\hline AEFES & 28 & 42 & 39 \\
\hline ANELE & 34 & 38 & 34 \\
\hline ARCLK & 30 & 22 & 18 \\
\hline ASELS & 11 & 18 & 21 \\
\hline AYGAZ & 1 & 10 & 2 \\
\hline BRISA & 39 & 20 & 32 \\
\hline CCOLA & 15 & 27 & 20 \\
\hline CIMSA & 40 & 44 & 44 \\
\hline DOHOL & 2 & 17 & 8 \\
\hline DOAS & 17 & 16 & 13 \\
\hline ENJSA & 37 & 26 & 26 \\
\hline ENKAI & 6 & 21 & 16 \\
\hline EREGL & 8 & 33 & 23 \\
\hline FROTO & 3 & 2 & 1 \\
\hline GLYHO & 41 & 41 & 43 \\
\hline SAHOL & 44 & 36 & 42 \\
\hline ISDMR & 4 & 12 & 6 \\
\hline KERVT & 14 & 6 & 5 \\
\hline KCHOL & 26 & 28 & 24 \\
\hline KORDS & 31 & 37 & 35 \\
\hline LOGO & 9 & 19 & 19 \\
\hline MGROS & 45 & 1 & 11 \\
\hline OTKAR & 16 & 3 & 3 \\
\hline PETKIM & 20 & 13 & 14 \\
\hline POLHO & 32 & 43 & 41 \\
\hline SODA & 7 & 7 & 7 \\
\hline TATGD & 5 & 14 & 9 \\
\hline TAVHL & 38 & 39 & 40 \\
\hline TKFEN & 12 & 11 & 15 \\
\hline TOASO & 13 & 4 & 4 \\
\hline TRKCM & 22 & 34 & 29 \\
\hline TCELL & 19 & 24 & 22 \\
\hline TUPRS & 23 & 23 & 17 \\
\hline THYAO & 33 & 35 & 33 \\
\hline TTKOM & 35 & 15 & 28 \\
\hline TTRAK & 27 & 9 & 10 \\
\hline SISE & 18 & 31 & 25 \\
\hline ULKER & 25 & 29 & 30 \\
\hline VESBE & 10 & 8 & 12 \\
\hline VESTL & 36 & 30 & 36 \\
\hline ZOREN & 42 & 5 & 38 \\
\hline
\end{tabular}

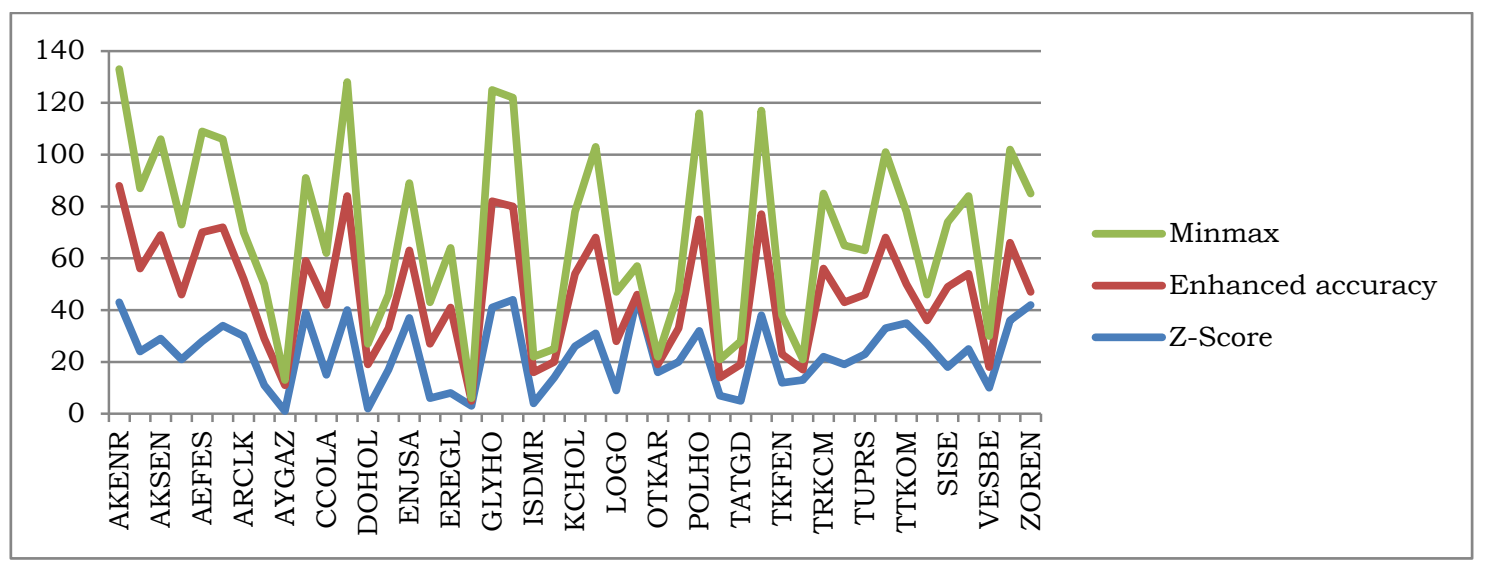

Figure 1. Ranking Results 
It is very difficult to decide which technique is the most suitable for the PIV method by using the data in Table 9 and Figure 1 enhanced accuracy and linear max-min normalization techniques were used instead of the vector normalization technique in the PIV methods' algorithm.

\section{A PROPOSED APPROACH FOR SELECTING THE BEST SUITABLE TECHNIQUE FOR THE PIV METHOD}

Determining the best technique for MCDM methods is not an easy process. The rankings obtained are also different due to the differences in the algorithms of the methods. There are various approaches developed in the literature to select the most appropriate technique for MCDM methods.

Celen (2014) suggested the Pearson correlation approach to choose the best normalization techniques. Chakraborty and Yeh (2009) developed a RCI approach that takes into account consistency between the ranking results. Vafaei et al. (2018) used Spearman's rank correlation (used by Wang and Luo, 2010) in addition to approaches of Celen's (2014) and Chakraborty and Yeh's (2009).

In this study, a four-stage model was proposed. In the first stage, the consistency between the ranking results was examined using the RCI approach (Chakraborty and Yeh, 2009). In the second stage, the relationship between the ranking results obtained with different techniques was examined using the Pearson correlation technique (Celen, 2014). In the third stage, new rankings were obtained using the STD approach from (Bland and Altman, 1996; Yeh, 2003). In the fourth step, Minkowski distances (Guo, 2004; Hassan et al. 2014) were calculated to determine the most appropriate technique for the PIV method.

Step A: Determining the RCI from Chakraborty and Yeh (2009).

Ranking consistency is used to show how well a normalization procedure produces similar rankings with other procedures. The higher the RCI, the better the procedure is (Chakraborty and Yeh, 2009, p.1817). In this study, the consistency weight (CW) was used as follows:

1) If a technique is consistent with all other two techniques, then $\mathrm{CW}=2 / 2=1$

2) If a technique is consistent with one of the two techniques, then $\mathrm{CW}=1 / 2$

3) If a technique is not consistent with any other five techniques, then $\mathrm{CW}=0 / 2=0$.

The ranking consistency index of $Z$-Score is calculated as;

$\mathrm{RCI}($ Vector $\left.)=\left[\left(\mathrm{T} 123^{*}(\mathrm{CW}=1)\right)+(\mathrm{T} 12 *(\mathrm{CW}=1 / 2))+\left(\mathrm{T} 13^{*}(\mathrm{CW}=1 / 2)\right)+\mathrm{TD} 123^{*}(\mathrm{CW}=0)\right) / \mathrm{TS}\right]$

The ranking consistency index of Enhanced accuracy is calculated as;

RCI (Vector) $\left.=\left[\left(\mathrm{T}_{123} *(\mathrm{CW}=1)\right)+\left(\mathrm{T}_{21} *(\mathrm{CW}=1 / 2)\right)+\left(\mathrm{T}_{23} *(\mathrm{CW}=1 / 2)\right)+\mathrm{TD}_{123} *(\mathrm{CW}=0)\right) / \mathrm{TS}\right]$

The ranking consistency index of $\mathbf{m i n}-\mathbf{m a x}$ is calculated as;

$\mathrm{RCI}($ Vector $\left.)=\left[\left(\mathrm{T}_{123} *(\mathrm{CW}=1)\right)+\left(\mathrm{T}_{31} *(\mathrm{CW}=1 / 2)\right)+\left(\mathrm{T}_{32} *(\mathrm{CW}=1 / 2)\right)+\mathrm{TD}_{123} *(\mathrm{CW}=0)\right) / \mathrm{TS}\right]$

where

RCI(X) RCI for normalization procedure (X $\left.=\mathrm{N}_{1}, \mathrm{~N}_{2}, \ldots, \mathrm{N}_{3}\right)$

$T S=$ Total number of times the simulation was run (in this study $\mathrm{TS}=1$ )

$T D_{123}=$ Total number of times N1, N2, N3 produced different rankings

$T_{123}=$ Total number of times N1, N2, N3 produced the same ranking

$T_{12}=$ Total number of times N1, N2 produced the same ranking

RCI values were calculated for all normalization techniques and the results are presented in Table 10 . 
Application of the PIV Method in the Presence of Negative Data:

An Empirical Example from a Real-World Case

Table 10. RCI Values and Ranking

\begin{tabular}{lrr}
\hline & RCI & Rank \\
\hline Z-Score & 4.5 & 3 \\
Enhanced accuracy & 6.0 & 2 \\
Min-max & 6.5 & 1 \\
\hline
\end{tabular}

From Table 10, the min-max normalization technique is the most suitable for the PIV method. This technique was followed by $Z$-score and enhanced accuracy techniques, respectively.

Step B: Determining the Pearson correlation from Celen (2014).

In this step, Pearson correlation coefficient was calculated using the ranking values in Table 9. The results obtained are presented in Table 11. The following formula (9) was used for calculating the Pearson correlation.

$r=\frac{\sum\left(x_{i}-\grave{x}\right)\left(y_{i}-\overleftrightarrow{y}\right)}{(N-1) \sigma_{x} \sigma_{y}}$

$x$ and $y$ indicate the arithmetic mean

$\mathrm{N}$ is the number of alternatives.

Table 11. Pearson Correlation Results and Mean Ks Values

\begin{tabular}{lrrrrr}
\hline & Z-Score & Enhanced accuracy & Min-max & Mean ks & Rank \\
\hline Z-Score & - & -0.100 & 0.687 & 0.294 & 2 \\
Enhanced accuracy & -0.100 & - & 0.594 & 0.247 & 3 \\
Min-max & 0.687 & 0.594 & - & 0.641 & 1 \\
\hline
\end{tabular}

According to the results in Table 11, the min-max normalization technique was determined to be the most suitable for the PIV method, while the enhanced accuracy normalization technique was determined to be the most inappropriate technique.

Step C: Calculation of STD from (Bland and Altman, 1996; Yeh, 2003).

The standard deviation was calculated using the data in Table 9 and the results obtained are presented in Table 12.

The STD formula is expressed as:

$S T D=\sqrt{\frac{\sum_{i=1}^{P}\left(x_{i}-\grave{x}\right) 2}{q-1}}$

Table 12. STD Results for the Different Techniques

\begin{tabular}{lrr}
\hline & STD & Rank \\
\hline Z-Score & 0.016 & 2 \\
\hline Enhanced accuracy & 0.006 & 3 \\
\hline Min-max & 0.069 & 1 \\
\hline
\end{tabular}

According to the results in Table 12, the min-max normalization technique was determined to be the most suitable for the PIV method, while the enhanced accuracy normalization technique was determined to be the least appropriate technique.

Step D: Calculating Minkowski Distances (Guo, 2004; Hassan et al. 2014)

In the last step, Minkowski distance measurements were used to determine the most suitable technique for the PIV method. Accordingly, the most used measurements such as Manhattan, Euclidean and Chebyshev were preferred in this study. The formulas of the methods are as shown in equations (11), (12) and (13) respectively.

$\begin{array}{ll}\text { Manhattan } & (p=1): d(x, y)=\sum_{i=1}^{n}\left|x_{i}-y_{i}\right| \\ \text { Euclidean } & (p=2): d(x, y)=\sqrt{\sum_{i=1}^{n}\left(x_{i}\right.}-y_{i) 2} \\ \text { Chebyshev } \quad(p=\infty): d(x, y)=\max _{i}\left(\left|x_{i}-y_{i}\right|\right)\end{array}$ 
Table 13. Minkowski Distances Measurement Results for the Normalization Methods

\begin{tabular}{lrrrrrr}
\hline & Manhattan & Rank & Euclidean & Rank & Chebishev & Rank \\
\hline Z-Score & 16.992 & 2 & 3.933 & 2 & 0.075 & 2 \\
\hline Enhanced accuracy & 4.676 & 3 & 1.022 & 3 & 0.039 & 3 \\
\hline Min-max & 78.214 & 1 & 17.599 & 1 & 0.342 & 1 \\
\hline
\end{tabular}

In this study, a four-step process was followed to determine the most suitable technique for the PIV method in the presence of negative values in the decision matrix. Except for RCI, ranking results obtained with Pearson correlation, STD, and Minkowski measurements were determined to be the same. According to Table 13, min-max is the most suitable technique for the PIV method. However, the plural voting method suggested by Vafaei et al. (2020) is used at this stage to reach a definite conclusion. Thus, the alternative with the highest number of first ranks is chosen. The results of plurality voting method are given in Table 14.

Table 14. The Final Ranking Results

\begin{tabular}{lrrrrrrr}
\hline & RCI & $\begin{array}{r}\text { Mean Ks } \\
\text { (Pearson) }\end{array}$ & STD & Manhattan & Euclidean & $\begin{array}{r}\text { Chebishev } \\
\text { Plurality } \\
\text { Voting }\end{array}$ \\
\hline Z-Score & 3 & 2 & 2 & 2 & 2 & 0 \\
\hline Enhanced accuracy & 2 & 3 & 3 & 3 & 3 & 0 \\
\hline Minmax & 1 & 1 & 1 & 1 & 1 & 1 \\
\hline
\end{tabular}

According to the results in Table 14, the most suitable technique for the PIV method is the minmax normalization technique. The Z-Score standardization technique and enhanced accuracy normalization technique were identified as the least appropriate techniques for the PIV method.

\section{CONCLUSION}

In MCDM methods, negative and zero value data are generally not found in the decision matrix. In such a case, it is necessary to convert the negative decision matrix data to positive because the values in the normalized decision matrix must be between 0 and 1 .

The PIV method cannot be used when there are negative values in the decision matrix. It is necessary to change the normalization method or standardize the decision matrix elements to obtain the final result with the PIV method. In this study, in which the financial performance of companies listed in the BİST sustainability index are evaluated, two different solutions have been proposed to the negative data problem in the PIV method and the most effective approach has been tried to be determined. Firstly, the decision matrix with negative data value was converted to positive using the $Z$-Score standardization method developed by Zhang et al. (2014), and the steps of the PIV method were applied. Then, the PIV method steps were repeated using the enhanced accuracy and min-max normalization techniques instead of the vector normalization technique included in the algorithm of the PIV method.

This study sought to answers the following research questions:

- In the presence of observations with negative values in the decision matrix, would it be more appropriate to standardize the decision matrix or change the normalization technique to use MCDM methods?

- If the second way is to be followed, which normalization technique is most suitable for the PIV method?

In this study, a four-step process was applied to find answers to the above questions and RCI values developed by Chakraborty and Yeh (2009) were calculated. In the second stage, the Pearson correlation values of the ranking results obtained by different techniques were calculated by following the approach suggested by Celen (2014). In the third step, the STD of the ranking values 
Application of the PIV Method in the Presence of Negative Data: An Empirical Example from a Real-World Case

was calculated (Bland and Altman, 1996; Yeh, 2003). In the fourth step, Manhattan, Euclidean, Chebyshev distance measures, (Guo, 2004; Hassan et al. 2014) were calculated. In the last stage, the plurality voting method (d'Angelo et al. 1998) proposed by Vafaei et al. (2020) was used to obtain a final result. Accordingly, the most suitable technique for the PIV method is the min-max normalization technique. The $Z$-score standardization technique and enhanced accuracy normalization techniques were determined unsuitable for the PIV method. As a result, the minmax normalization technique is the best solution to use for the PIV method in the presence of negative data values.

This study is helpful for researchers who want to use the PIV method in presence of negative values in the decision matrix and motivates them for future studies. In addition, this study guides researchers to use other MCDM techniques when there are negative values in the decision matrix. In future studies, the proposed model can be tested for different MCDM methods and the number of normalization techniques can be increased.

Peer-review: Externally peer-reviewed

Conflict of Interest: The authors declare that there is no conflict of interest.

Funding: The authors received no financial support for the research, authorship and/or publication of this article.

Ethical Approval: This article does not contain any studies with human participants or animals performed by the author.

Author Contributions: Nazlı Ersoy (100\%)

\section{REFERENCES}

Abdel-Basset, M., Ding, W., Mohamed, R., and Metawa, N. (2020). An integrated plithogenic MCDM approach for financial performance evaluation of manufacturing industries. Risk Management, 22(3), 192-218. doi:10.1057/s41283-020-00061-4

Asgharpour, M. J. (1998). Multiple criteria decision making. Tehran: Tehran University Press.

Bland, J. M., and Altman, D.G. (1996). Statistics notes: measurement error. BMJ, 313(7059), 744744. doi: $10.1136 /$ bmj.313.7059.744

Celen, A. (2014). Comparative analysis of normalization procedures in TOPSIS method: with an application to Turkish deposit banking market. Informatica, 25(2), 185-208. doi: 10.15388/Informatica.2014.10

Chakraborty, S., and Yeh, C. H. (2007). A Simulation Based Comparative Study of Normalization Procedures in Multiattribute Decision Making. In Proceedings of the 6th Conference on 6th WSEAS Int. Conference on Artificial Intelligence, Knowledge Engineering and Data Bases (p. 102-109). Wisconsin, USA.

Chakraborty, S., and Yeh, C. H. (2009). A Simulation Comparison of Normalization Procedures for TOPSIS. In 2009 International Conference on Computers and Industrial Engineering (p. 1815-1820). France.

Chatterjee, P., and Chakraborty, S. (2014). Investigating the effect of normalization norms in flexible manufacturing sytem selection using multi-criteria decision-making methods. Journal of Engineering Science and Technology Review, 7(3), 141-150. doi:10.25103/jestr.073.23 
d'Angelo, A., Eskandari, A., and Szidarovszky, F. (1998). Social choice procedures in water resource management. Journal of Environmental Management, 52(3), 203-210. doi: 10.1006/jema.1997.0156

Dinçer, H., and Yüksel, S. (2018). Comparative evaluation of BSC-based new service development competencies in Turkish banking sector with the integrated fuzzy hybrid MCDM using content analysis. International Journal of Fuzzy Systems, 20(8), 2497-2516. doi: $10.1007 / \mathrm{s} 40815-018-0519-y$

Ehrgott, M., Klamroth, K., and Schwehm, C. (2004). An MCDM approach to portfolio optimization. European Journal of Operational Research, 155(3), 752-770. doi: 10.1016/S0377-2217(02)00881-0

Farag, M. M. (1997). Materials selection for engineering design. USA: Prentice Hall.

Ghadikolaei, S. A., Esbouei, K. S., and Antucheviciene, J. (2014). Applying fuzzy MCDM for financial performance evaluation of Iranian companies. Technological and Economic Development of Economy, 20(2), 274-291. doi: 10.3846/20294913.2014.913274

Guo, Q. (2004). Minkowski Measure of Asymmetry and Minkowski Distance for Convex Bodies. Doctoral Dissertation. Uppsala University Department of Mathematics, Uppsala.

Hassan, D., Aickelin, U., and Wagner, C. (2014). Comparison of Distance Metrics for Hierarchical Data in Medical Databases. International Joint Conference on Neural Networks (p. 36363643). Beijing, China.

Jahan, A., and Edwards, K.L. (2015). A state-of-the-art survey on the influence of normalization techniques in ranking: improving the materials selection process in engineering design. Materials and Design, 65, 335-342. doi: 10.1016/j.matdes.2014.09.022

Jahan, A., Mustapha, F., Sapuan, S. M., Ismail, M. Y., and Bahraminasab, M. (2012). A framework for weighting of criteriain ranking stage of material selection process. The International Journal of Advanced Manufacturing Technology, 58(1), 411-420. doi: 10.1007/s00170-0113366-7

Jüttler, H. (1966). Untersuchungen zur Fragen der Operationsforschung und ihrer Anwendungsmöglichkeiten auf ökonomische Problemstellungen unter besonderer Berücksichtigung der Spieltheorie. Dissertation A an der Wirtschaftswissenschaftlichen Fakultät der Humboldt-Universität Berlin.

Kosareva, N., Krylovas, A., and Zavadskas, E. K. (2018). Statistical analysis of MCDM data normalization methods using monte carlo approach: the case of ternary estimates matrix. Economic Computation and Economic Cybernetics Studies and Research, 52, 159175. doi: 10.24818/18423264/52.4.18.11

Körth, H. (1969). Zur Berücksichtigung mehrer Zielfunktionen bei der Optimierung von Produktionsplanen. Mathematik und Wirtschaft, 6, 184-201.

Kumar, A., Sah, B., Singh, A. R., Deng, Y., He, X., Kumar, P., and Bansal, R. C. (2017). A review of multi criteria decision making (MCDM) towards sustainable renewable energy development. Renewable and Sustainable Energy Reviews, 69, 596-609. doi: 10.1016/j.rser.2016.11.191

Lai, Y.J., and Hwang, C.L. (1994). Fuzzy multiple objective decision making: methods and applications. Berlin: Springer. 
Application of the PIV Method in the Presence of Negative Data: An Empirical Example from a Real-World Case

Markovic, Z. (2010). Modification of TOPSIS method for solving of multicriteria tasks. The Yugoslav Journal of Operations Research, 20(1), 117-143. doi: 10.2298/YJOR1001117M

Mathew, M., Sahu, S., and Upadhyay, A. K. (2017). Effect of normalization techniques in robot selection using weighted aggregated sum product assessment. Int. J. Innov. Res. Adv. Stud, 4(2), 59-63. Retrieved from: https://www.ijiras.com/2017/Vol_4Issue_2/paper_12.pdf

Matić, B., Jovanović, S., Das, D. K., Zavadskas, E. K., Stević, Ž., Sremac, S., and Marinković, M. (2019). A new hybrid MCDM model: sustainable supplier selection in a construction company. Symmetry, 11(3), 1-24. doi: 10.3390/sym11030353

Milani, A. S., Shanian, A., Madoliat, R., and Nemes, J. A. (2005). The effect of normalization norms in multiple attribute decision making models: a case study in gear material selection. Structural and Multidisciplinary Optimization, 29(4), 312-318. doi: 10.1007/s00158-0040473-1

Mousavi-Nasab, S. H., and Sotoudeh-Anvari, A. (2017). A comprehensive MCDM-based approach using TOPSIS, COPRAS and DEA as an auxiliary tool for material selection problems. Materials and Design, 121, 237-253. doi: 10.1016/j.matdes.2017.02.041

Mufazzal, S., and Muzakkir, S. M. (2018). A new multi-criterion decision making (MCDM) method based on proximity indexed value for minimizing rank reversals. Computers and Industrial Engineering, 119, 427-438. doi: 10.1016/j.cie.2018.03.045

Peldschus, F. (1986). Zur Anwendung der Theorie der Spiele für Aufgaben der Bautechnologie. Dissertation B, Technische Hochschule Leipzig.

Pineda, P. J. G., Liou, J. J., Hsu, C. C., and Chuang, Y. C. (2018). An integrated MCDM model for improving airline operational and financial performance. Journal of Air Transport Management, 68, 103-117. doi: 10.1016/j.jairtraman.2017.06.003

Shyur, H. J., and Shih, H. S. (2006). A hybrid MCDM model for strategic vendor selection. Mathematical and Computer Modelling, 44(7-8), 749-761. doi: 10.1016/j.mcm.2005.04.018

Stević, Ž., Pamučar, D., Puška, A., and Chatterjee, P. (2020). Sustainable supplier selection in healthcare industries using a new MCDM method: measurement of alternatives and ranking according to compromise solution (MARCOS). Computers and Industrial Engineering, 140, 1-15. doi: 10.1016/j.cie.2019.106231

Stopp, F. (1975). Variantenvergleich durch matrixspiele, Wissenschaftliche Zeitschrift der Hochschule für Bauwesen Leipzig, 2(117).

Tzeng, G. H., and Huang, J. J. (2011). Multiple attribute decision making: methods and applications, Florida, ABD: CRC Press.

Vafaei, N., Ribeiro, R. A., and Camarinha-Matos, L. M. (2016). Normalization Techniques for MultiCriteria Decision Making: Analytical Hierarchy Process Case Study. In Doctoral Conference on Computing, Electrical and Industrial Systems (p. 261-269). Springer, Cham.

Vafaei, N., Ribeiro, R. A., and Camarinha-Matos, L. M. (2018). Data normalisation techniques in decision making: case study with TOPSIS method. International Journal of Information and Decision Sciences, 10(1), 19-38. doi: 10.1504/IJIDS.2018.090667 
Vafaei, N., Ribeiro, R. A., and Camarinha-Matos, L. M. (2020). Selecting Normalization Techniques for the Analytical Hierarchy Process. In Doctoral Conference on Computing, Electrical and Industrial Systems (p. 43-52). Springer, Cham.

Vafaei, N., Ribeiro, R. A., Camarinha-Matos, L. M., and Valera, L. R. (2019). Normalization techniques for collaborative networks. Kybernetes, 49(4), 1285-1304. doi: 10.1108/K-092018-0476

Wang, Y. M., and Luo, Y. (2010). Integration of correlations with standard deviations for determining attribute weights in multiple attribute decision making. Mathematical and Computer Modelling, 51(1-2), 1-12. doi: 10.1016/j.mcm.2009.07.016

Weitendorf, D. (1976). Beitrag zur Optimierung der räumlichen Struktur eines Gebäudes, Dissertation A. Hochschule für Architektur und Bauwesen. Weimar.

Yazdani, M., Chatterjee, P., Zavadskas, E. K., and Zolfani, S. H. (2017b). Integrated QFD-MCDM framework for green supplier selection. Journal of Cleaner Production, 142, 3728-3740. doi: $10.1016 /$ j.jclepro.2016.10.095

Yazdani, M., Jahan, A., and Zavadskas, E. (2017a). Analysis in material selection: influence of normalization tools on copras-g. Economic Computation and Economic Cybernetics Studies and Research, 51(1), 59-74. Retrieved from: http://www.ipe.ro/RePEc/cys/ecocyb_pdf/ecocyb1_2017p59-74.pdf

Yeh, C. H. (2003). The selection of multiattribute decision making methods for scholarship student selection. International Journal of Selection and Assessment, 11(4), 289-296. doi: 10.1111/j.0965-075X.2003.00252.x

Zavadskas, E. K., and Turskis, Z. (2008). A new logarithmic normalization method in games theory. Informatica, 19(2), 303-314. doi: 10.15388/Informatica.2008.215

Zhang, X., Wang, C., Li, E., and Xu, C. (2014). Assessment model of eco-environmental vulnerability based on 1mproved entropy weight method. The Scientific World Journal, 2014, 1-7. doi: 10.1155/2014/797814 
Application of the PIV Method in the Presence of Negative Data: An Empirical Example from a Real-World Case

\section{APPENDIX}

\section{Appendix 1. Alternatives}

\begin{tabular}{|c|c|c|c|c|c|}
\hline Rank & Company's name & Rank & Company's name & Rank & Company's name \\
\hline 1 & $\begin{array}{l}\text { AKENERJİ ELEKTRIKK } \\
\text { ÜRETIM A.Ş. (AKENR) }\end{array}$ & 16 & ENKA İNŞAAT VE SANAYİ A.Ş. (ENKAI) & 31 & $\begin{array}{l}\text { TAT GIDA SANAYİ A.Ş. } \\
\text { (TATGD) }\end{array}$ \\
\hline 2 & $\begin{array}{l}\text { AKSA AKRİL̇K KİMYA } \\
\text { SANAYİI A.Ş. (AKSA) }\end{array}$ & 17 & $\begin{array}{l}\text { EREĞLİ DEMİR VE ÇELİK FABRİKALARI } \\
\text { T.A.Ş. (EREGL) }\end{array}$ & 32 & $\begin{array}{l}\text { TAV HAVALİMANLARI } \\
\text { HOLDING A.Ș. (TAVHL) }\end{array}$ \\
\hline 3 & $\begin{array}{l}\text { AKSA ENERJİ ÜRETIM A.Ş. } \\
\text { (AKSEN) }\end{array}$ & 18 & FORD OTOMOTIV SANAYİ A.Ş. (FROTO) & 33 & $\begin{array}{l}\text { TEKFEN HOLDING A.Ş. } \\
\text { (TKFEN) }\end{array}$ \\
\hline 4 & $\begin{array}{l}\text { ANADOLU CAM SANAYIII A.Ş. } \\
\text { (ANACM) }\end{array}$ & 19 & $\begin{array}{l}\text { GLOBAL YATIRIM HOLDING A.Ş. } \\
\text { (GYLHO) }\end{array}$ & 34 & $\begin{array}{l}\text { TOFAS TÜRK OTOMOBİL } \\
\text { FABRİKASI A.Ş. (TOASO) }\end{array}$ \\
\hline 5 & $\begin{array}{l}\text { ANADOLU EFES BİRACILIK } \\
\text { VE MALT SANAYİI A.Ş. } \\
\text { (AEFES) }\end{array}$ & 20 & $\begin{array}{l}\text { HACI ÖMER SABANCI HOLDİNG A.Ş. } \\
\text { (SAHOL) }\end{array}$ & 35 & $\begin{array}{l}\text { TRAKYA CAM SANAYİI A.Ş. } \\
\text { (TRKCM) }\end{array}$ \\
\hline 6 & $\begin{array}{l}\text { ANEL ELEKTRİK PROJE } \\
\text { TAAHHÜT VE TİCARET A.Ş. } \\
\text { (ANELE) }\end{array}$ & 21 & $\begin{array}{l}\text { İSKENDERUN DEMİR VE ÇELİK A.Ş. } \\
\text { (ISDMR) }\end{array}$ & 36 & $\begin{array}{l}\text { TURKCELL İLETIŞIM } \\
\text { HİZMETLERİ A.Ş. (TCELL) }\end{array}$ \\
\hline 7 & ARÇELİK A.Ş. (ARCLK) & 22 & $\begin{array}{l}\text { KEREVITASS GIDA SANAYİ VE TİCARET } \\
\text { A.Ş. (KERVT) }\end{array}$ & 37 & $\begin{array}{l}\text { TÜPRAŞ-TÜRKIYY PETROL } \\
\text { RAFİNERİLERİ A.Ş. (TUPRS) }\end{array}$ \\
\hline 8 & $\begin{array}{l}\text { ASELSAN ELEKTRONIK } \\
\text { SANAYİ VE TICARET A.Ş. } \\
\text { (ASELS) }\end{array}$ & 23 & KOÇ HOLDİNG A.Ş. (KCHOL) & 38 & $\begin{array}{l}\text { TÜRK HAVA YOLLARI A.O. } \\
\text { (THYAO) }\end{array}$ \\
\hline 9 & AYGAZ A.Ş. (AYGAZ) & 24 & KORDSA TEKNIK TEKSTİL A.Ş. (KORDS) & 39 & $\begin{array}{l}\text { TÜRK } \\
\text { TELEKOMÜNİKASYON A.Ş. } \\
\text { (TTKOM) }\end{array}$ \\
\hline 10 & $\begin{array}{l}\text { BRISAA BRIDGESTONE } \\
\text { SABANCI LASTIK SANAYİ VE } \\
\text { TİCARET A.Ş. (BRISA) }\end{array}$ & 25 & $\begin{array}{l}\text { LOGO YAZILIM SANAYİ VE TİCARET A.Ş. } \\
\text { (LOGO) }\end{array}$ & 40 & $\begin{array}{l}\text { TÜRK TRAKTÖR VE ZİRAAT } \\
\text { MAKINELERİ A.Ş. (TTRAK) }\end{array}$ \\
\hline 11 & $\begin{array}{l}\text { COCA-COLA İÇECEK A.S.S. } \\
\text { (CCOLA) }\end{array}$ & 26 & MİGROS TİCARET A.Ş. (MIGROS) & 41 & $\begin{array}{l}\text { TÜRKIYE SİSE VE CAM } \\
\text { FABRIKALARI A.Ş. (SISE) }\end{array}$ \\
\hline 12 & $\begin{array}{l}\text { ÇIMSSA ĊIMENTO SANAYİ VE } \\
\text { TİCARET A.Ş. (CIMSA) }\end{array}$ & 27 & $\begin{array}{l}\text { OTOKAR OTOMOTIV VE SAVUNMA } \\
\text { SANAYI A.Ş (OTKAR) }\end{array}$ & 42 & $\begin{array}{l}\text { ÜLKER BISKÜVİ SANAYİ } \\
\text { A.Ş. (ULKER) }\end{array}$ \\
\hline 13 & $\begin{array}{l}\text { DOĞAN ŞİRKETLER GRUBU } \\
\text { HOLDING A.Ş. (DOHOL) }\end{array}$ & 28 & $\begin{array}{l}\text { PETKIM PETROKİMYA HOLDİNG A.Ş. } \\
\text { (PETKIM) }\end{array}$ & 43 & $\begin{array}{l}\text { VESTEL BEYAZ ESYYA } \\
\text { SANAYI VE TİCARET A.Ş. } \\
\text { (VESBE) }\end{array}$ \\
\hline 14 & $\begin{array}{l}\text { DOĞUŞ OTOMOTIV SERVİS } \\
\text { VE TİCARET A.Ş. (DOAS) }\end{array}$ & 29 & POLİSAN HOLDİNG A.Ş. (POLHO) & 44 & $\begin{array}{l}\text { VESTEL ELEKTRONIK } \\
\text { SANAYI VE TÍCARET A.Ş. } \\
\text { (VESTL) }\end{array}$ \\
\hline 15 & $\begin{array}{l}\text { ENERJİSA ENERJİ A.Ş. } \\
\text { (ENJSA) }\end{array}$ & 30 & SODA SANAYİI A.Ş. (SODA) & 45 & $\begin{array}{l}\text { ZORLU ENERJİ ELEKTRİK } \\
\text { ÜRETIM A.Ş. (ZOREN) }\end{array}$ \\
\hline
\end{tabular}




\section{GENIŞLETİLMIŞ ÖZET}

\section{Amaç}

Karar matrisinde negatif değerlerin varlığına Çok Kriterli Karar Verme (ÇKKV) yöntemlerinde nadir olarak rastlanır. Böyle bir durumda ise karar matrisinde yer alan negatif değerler pekçok ÇKKV yönteminde normalize matriste negatif değerlere yol açmaktadır ve işleme devam edilememektedir. Proximity Indexed Value (PIV) yöntemi de işleme devam edilemeyen yöntemler arasında yer almaktadır. Bu çalışmanın amacı, karar matrisinde negatif verilerin varlığ durumunda PIV yöntemini uygulayabilmek için en iyi çözüm yolunu sunmaktır.

\section{Yöntem}

Negatif verilerin varlığı durumunda PIV yöntemini kullanabilmek amacıyla iki farklı çözüm yolu sunulmuştur. İlk olarak, negatif karar matrisi elemanları, Z-skor standartlaştırma yöntemi kullanılarak pozitif hale getirilmiştir. İkinci yol olarak, PIV yönteminin algoritmasında bulunan vektör normalizasyon tekniği yerine Gelişmiş doğruluk (Enhanced accuracy) ve min-max normalizasyon teknikleri kullanılmıştır. En iyi çözüm yolunu seçmek amacıyla ise dört aşamalı bir yaklaşım önerilmiştir. İlk aşamada, Tutarlılık İndeksi (Ranking Consistency Index (RCI)) kullanılmış ve sıralama tutarlılığı hesaplanmıştır. İkinci aşamada, Pearson korelasyon katsayısı hesaplanmıştır. Üçüncü aşamada, standart sapma (STD) yaklaşımı ele alınmıştır. Dördüncü aşamada, Minkowski uzaklık ölçülerinden Manhattan, Euclidean, Chebyshev ölçüleri hesaplanmıştır. Son aşamada ise çoğul oy sistemi (Plural voting) yöntemi kullanılarak en iyi yaklaşım belirlenmiştir.

\section{Bulgular}

Elde edilen sonuçlara gore karar matrisinde negatif verilerin varlığı durumunda PIV yöntemini kullanabilmek için en iyi yol normalizasyon yöntemini değiştirmek ve min-max normalizasyon tekniğini kullanmaktır. Z-skor standartlaştırma tekniği ve Gelişmiş doğruluk (Enhanced accuracy) normalizasyon tekniği, min-max normalizasyon tekniğine kiyasla daha az etkin yöntemler olarak tespit edilmiştir.

\section{Sonuç}

ÇKKV yöntemlerinde, karar matrisinde genellikle negatif ve sıfır değerli veriler bulunmaz. Böyle bir durumda normalize edilmiş karar matrisindeki değerlerin 0 ile 1 arasında olması gerektiğinden negatif karar matrisi verilerini pozitife çevirmek gerekir.

Karar matrisinde negatif değerler olduğunda PIV yöntemi kullanılamaz. PIV yöntemi ile nihai sonucu elde etmek için normalizasyon yöntemini değiştirmek veya karar matrisi elemanlarını standardize etmek gerekir. Borsa İstanbul (BİST) sürdürülebilirlik endeksinde yer alan şirketlerin finansal performanslarının değerlendirildiği bu çalışmada, PIV yöntemindeki olumsuz veri sorununa iki farklı çözüm önerilmiş ve en etkin yaklaşım belirlenmeye çalışılmıştır. İlk olarak, negatif verilere sahip olan karar matrisi, Z-Skor standartlaştırma yöntemi kullanılarak pozitife dönüştürülmüştür. Daha sonra, PIV yönteminin algoritmasinda yer alan vektör normalizasyon tekniği yerine gelişmiş doğruluk (Enhanced accuracy) ve min-max normalizasyon teknikleri kullanılarak PIV yöntemi adımları tekrarlanmıştır.

Bu çalışmada aşağıdaki araştırma sorularına yanıt aranmıştır:

- Karar matrisinde negatif gözlem değerlerinin varlığında ÇKKV yöntemlerini kullanabilmek için karar matrisini standartlaştırmak mı yoksa normalleştirme tekniğini değiştirmek mi daha uygun olur? 
Application of the PIV Method in the Presence of Negative Data: An Empirical Example from a Real-World Case

- İkinci yol izlenecek ise PIV yöntemi için en uygun normalizasyon tekniği hangisidir?

Bu çalışmada yukarıdaki sorulara cevap bulmak için dört aşamalı bir süreç uygulanmıştır. İlk aşamada RCI değerleri hesaplanmıştır. İkinci aşamada, farklı tekniklerle elde edilen sıralama sonuçlarının Pearson korelasyon değerleri hesaplanmıştır. Üçüncü aşamada, sıralama değerlerinin STD'si hesaplanmıştır. Dördüncü aşamada, Manhattan, Euclidean, Chebyshev uzaklık ölçüleri hesaplanmıştır. Son aşamada, çoğul oylama yöntemi nihai bir sonuç elde etmek için kullanılmıştır. Buna göre PIV yöntemi için en uygun teknik min-max normalizasyon tekniği olarak tespit edilmiştir. Z-skor standartlaştırma ve gelişmiş doğruluk (Enhanced accuracy) normalizasyon tekniklerinin PIV yöntemi için uygun olmadiğı belirlenmiştir. Sonuç olarak, normalizasyon tekniği değişimine giderek min-max normalizasyon tekniğini kullanmak, negatif verilerin varlığında PIV yöntemini kullanmak için en iyi çözümdür.

$\mathrm{Bu}$ çalışma, karar matrisinde negatif değerlerin varlığında PIV yöntemini kullanmak isteyen araştırmacılara yardımcı olmakta ve onları gelecek çalışmalar için motive etmektedir. Ayrıca bu çalışma, karar matrisinde negatif değerler olduğunda araştırmacılara diğer ÇKKV tekniklerini kullanmaları konusunda rehberlik etmektedir. 